\title{
Radiation-Induced Leiomyosarcoma of the Prostate after Brachytherapy for Prostatic Adenocarcinoma
}

\author{
Hiroto Horiguchi ${ }^{a, d} \quad K^{\text {Kohichi Takada }}{ }^{a}$ Yusuke Kamihara $^{a}$ Soushi Ibata ${ }^{a}$ \\ Satoshi Iyama ${ }^{\mathrm{a}}$ Tsutomu Sato $^{\mathrm{a}}$ Tsuyoshi Hayashi $^{\mathrm{a}}$ Koji Miyanishi ${ }^{\mathrm{a}}$ \\ Yasushi Sato $^{a}$ Rishu Takimoto ${ }^{a}$ Masayoshi Kobune ${ }^{a}$ Ko Kobayashi ${ }^{b}$ \\ Yasuo Hirayama $^{d}$ Naoya Masumori $^{\mathrm{b}}$ Tadashi Hasegawa $^{c}$ Junji Kato ${ }^{a}$ \\ Departments of ${ }^{a}$ Medical Oncology and Hematology, ${ }^{b}$ Urological Surgery and Andrology, \\ and 'Surgical Pathology, Sapporo Medical University School of Medicine, and \\ ${ }^{\mathrm{d}}$ Department of Internal Medicine, Higashi Sapporo Hospital, Sapporo, Japan
}

\section{Key Words}

Radiation-induced sarcoma $\cdot$ Brachytherapy $\cdot$ Leiomyosarcoma $\cdot$ Prostatic sarcoma $\cdot$ p53

\begin{abstract}
Radiation therapy (RTx) has been employed as a curative therapy for prostatic adenocarcinoma. RTx-induced sarcomas (RISs) are rare, late adverse events, representing less than $0.2 \%$ of all irradiated patients. RISs are more aggressive tumors than prostatic adenocarcinomas. Herein, we present a case with RTx-induced prostatic leiomyosarcoma after permanent brachytherapy for prostatic adenocarcinoma. A 69-year-old male presented with dysuria and gross hematuria. Six years previously, he had been diagnosed with localized prostate cancer and was treated by permanent brachytherapy. Urethroscopy showed stenosis by a tumor at the prostate. Transurethral prostatectomy was performed for a diagnosis. Based on pathological findings, the diagnosis was leiomyosarcoma of the prostate. He was treated with three cycles of neoadjuvant chemotherapy (CTx) that consisted of doxorubicin and ifosfamide (AI), followed by a prostatocystectomy with intrapelvic lymphadenectomy. The tumor extended from the prostate and infiltrated the bladder wall and serosa with lymphatic and venous invasion. The surgical margin was negative, and no residual prostatic adenocarcinoma was observed. The proportion of necrotic tumor cells by neoadjuvant CTx was around $50 \%$. Subsequently, adjuvant CTx was offered, but the patient chose a follow-up without CTx. Local recurrence and lung metastasis were detected by computed tomography 3 months after the surgery. He was treated again with AI. However, CTx was not effective and he died 6
\end{abstract}

Kohichi Takada, MD, PhD

Department of Medical Oncology and Hematology

Sapporo Medical University School of Medicine

South 1 West 16, Chuo-Ku, Sapporo 060-8543 (Japan)

E-Mail ktakada@sapmed.ac.jp 
Horiguchi et al.: Radiation-Induced Leiomyosarcoma of the Prostate after Brachytherapy for Prostatic Adenocarcinoma

months after the operation. In conclusion, an effective treatment strategy for prostatic sarcoma should be developed in the near future, although the clinical feature of prostatic sarcoma remains unclear due to its rare incidence.

(C) 2014 S. Karger AG, Basel

\section{Introduction}

Radiation therapy (RTx) has been employed as radical therapy as well as palliative therapy in the treatment of malignancies. Recently, RTx has been increasingly used as one of the curative treatments for localized prostatic adenocarcinoma [1]. Permanent brachytherapy is used for patients with low risk prostate cancer, and is the optimal therapeutic tool to maximize cancer control while minimizing morbidity [2].

RTx-induced sarcoma (RIS) is a rare, late adverse event, representing less than $0.2 \%$ of all irradiated patients $[3,4]$. RISs are mostly associated with external-beam radiation [4]. RTx-induced prostatic leiomyosarcoma after permanent brachytherapy is extraordinarily rare. Herein, we present a case of RTx-induced prostatic leiomyosarcoma after permanent brachytherapy for prostatic adenocarcinoma.

\section{Case Presentation}

A 69-year-old man presented with dysuria and gross hematuria. He was diagnosed 6 years ago with localized prostate cancer with a Gleason score of 6 . He was treated by permanent brachytherapy using ${ }^{125}$ Iodine (125I) with a planned total dose of $145 \mathrm{~Gy}$. Serum prostate-specific antigen was $9.5 \mathrm{ng} / \mathrm{ml}$ before treatment, but was normal after brachytherapy. Urethroscopy showed stenosis by a tumor at the prostate. Transurethral prostatectomy revealed that the tumor was comprised of smooth muscle with spindle cells that had cigar-shaped nuclei with prominent cellular atypia, and proliferated cells forming fascicles in a storiform pattern. Immunohistochemical staining was positive for alpha-smooth muscle actin (SMA) and vimentin, and negative for desmin, CD34, estrogen receptor, and progesterone receptor. Based on these findings, the diagnosis was leiomyosarcoma of the prostate. Computed tomography showed that the prostatic tumor was over $5 \mathrm{~cm}$ with a high density of metallic seeds, and had invaded directly to the inferior bladder wall (fig. 1a). There was no metastatic lesion. Positron emission tomography showed that the abnormal increased uptake was consistent with prostatic tumor (fig. 1b). Distant metastasis was not found. He was treated with three cycles of neoadjuvant chemotherapy (CTx) that consisted of doxorubicin and ifosfamide (AI) (doxorubicin $30 \mathrm{mg} / \mathrm{m}^{2}$ on days $1-2$, ifosfamide 2,000 $\mathrm{mg} / \mathrm{m}^{2}$ on days $1-5$, every 21 days), and then a prostatocystectomy was performed with intrapelvic lymphadenectomy. Pathological findings revealed that the spindle-shaped tumor cells formed fascicles with strongly atypical clubbed nuclei and acidophilic cytoplasm (fig. $2 \mathrm{a}, \mathrm{b}$ ). There were large polymorphic tumor cells with many mitoses (over 20/10 high power fields) and necrosis. The proportion of necrotic tumor cells by neoadjuvant CTx was about 50\%. The tumor extended from the prostate and infiltrated the bladder wall and serosa with lymphatic and venous invasion. The surgical margin was negative, and no residual prostatic adenocarcinoma was observed. Notably, nontumor prostate cells had enlarged as well as atypical nuclei due to RTx. Immunohistochemistry showed that tumor cells were positive for SMA (fig. 2c), vimentin (fig. 2d), muscle specific actin (HHF35), and desmin (fig. 2e) and focally positive for cytokeratin AE1/AE3, and negative for S-100 (fig. 2f), CD117 (fig. 2g), epithelial membrane antigen, and CD34 (fig. 2h). The Ki-67 labeling index 
Horiguchi et al.: Radiation-Induced Leiomyosarcoma of the Prostate after Brachytherapy for Prostatic Adenocarcinoma

was $60 \%$. Interestingly, immunohistochemical studies showed p53 overexpression in the nuclei of leiomyosarcoma cells (fig. 2i).

Subsequently, adjuvant CTx was offered to the patient because of a high possibility of recurrence, but he chose a follow-up without CTx. Local recurrence and lung metastasis were detected by computed tomography 3 months after the surgery. He was treated again with AI. However, CTx was not effective and he died 6 months after the operation.

\section{Discussion}

RISs are quite rare but constitute serious late adverse events in adults [3, 5]. RISs are thought to develop after a latent period of 3-17 years, and are associated with externalbeam radiation [5-7]. The criteria for diagnosis of RISs are [8]: (1) the patient had received prior radiation; (2) the neoplasm occurred within the radiation volume; (3) a latent period of some years had elapsed; (4) there was histologic or radiographic evidence for a preexisting condition, in addition to microscopic proof for sarcoma, and (5) cancer syndromes such as Li-Fraumeni or Rothmund-Thomson have been excluded. Our patient fulfilled four of the five preceding criteria. He was not tested for genetic predisposing syndrome because his familial history did not suggest a potential hereditary genetic disorder.

Prostatic leiomyosarcoma is an extremely rare neoplasm that accounts for less than $0.1 \%$ of primary prostatic malignancies [9]. The standard therapy for cure is surgery among resectable patients [10-13]. In terms of neoadjuvant and adjuvant CTx, the efficacies for leiomyosarcoma are disputed. However, most patients with metastasis or recurrence received anthracycline (doxorubicin or epirubicin)-based combinations with alkylating agents (ifosfamide, cyclophosphamide, or dacarbazine) and/or vinca alkaloids (vincristine or vinblastine) $[10,14]$. The clinical outcomes of patients with prostate leiomyosarcoma have been dismal. Vandoros et al. [13] reported that median survival was estimated at 17 months. Metastatic disease at presentation and positive surgical margins are associated with worse survival $[10,13]$. Moreover, RISs were associated with a poorer disease-specific survival than sporadic soft tissue sarcomas [5]. Therefore, we treated the patient with three cycles of neoadjuvant CTx that consisted of AI, which has been effective on leiomyosarcoma [15]. Pathological examination revealed that the proportion of necrotic tumor cells was around $50 \%$, suggesting that the tumor cells in our case were sensitive to AI.

Although the majority of cases have occurred after external beam radiotherapy $[3,16]$, only two previous cases of radiation-induced prostatic sarcoma after permanent brachytherapy are known $[17,18]$. The latent periods were 8 and 9 years, and total RTx doses were 270 and $145 \mathrm{~Gy}$, respectively. The latency in our case was 6 years, and the RTx dose was 145 Gy. These parameters were consistent with previously described cases. Gladdy et al. [5] reported that the latency (median, 23.5 years; range, 7-74) which was observed in external beam radiation-related leiomyosarcomas, was much longer than those of a different histology. However, the latencies of the three brachytherapy-related cases (including our case) were much shorter. This suggested that permanent brachytherapy might induce sarcomas earlier than external-beam radiation because of the higher dose to the prostate. The risk of RIS due to permanent brachytherapy should be investigated in future studies that involve a large cohort of patients.

Recently, Gonin-Laurent et al. [19] demonstrated that the rate of $p 53$ mutation in RISs (58\%) was significantly higher than that in sporadic sarcomas (16.8\%). They claimed that the inactivation of p53 in RISs is a consequence of the irradiation, and furthermore, p53 inactivation was an early trigger event in RISs [20]. In our case, leiomyosarcoma cells were 


\begin{tabular}{l|l}
\hline DOI: $10.1159 / 000366294$ & $\begin{array}{l}\text { C 2014 S. Karger AG, Basel } \\
\text { www.karger.com/cro }\end{array}$ \\
\hline
\end{tabular}

Horiguchi et al.: Radiation-Induced Leiomyosarcoma of the Prostate after Brachytherapy for Prostatic Adenocarcinoma

positive for p53 staining. This suggested that p53 inactivation might be associated with the onset of this radiation-induced leiomyosarcoma.

In conclusion, we described an extremely rare case of RTx-induced prostatic leiomyosarcoma after permanent brachytherapy for prostatic adenocarcinoma. A significant increase has been noted in the number of long-term cancer survivors with recent advances in radiotherapy. Therefore, it is important to employ careful long-term surveillance for patients treated with RTx. In addition, effective treatment strategies for prostatic sarcoma need to be developed.

\section{Disclosure Statement}

The authors have no conflicts of interest to declare.

\section{References}

1 National Comprehensive Cancer Network (NCCN): NCCN Clinical Practice Guidelines in Oncology. Prostate Cancer. Version 2.2014. Fort Washington, NCCN, 2014.

http://www.nccn.org/professionals/physician_gls/pdf/prostate.pdf (accessed May 23, 2014).

2 Koukourakis G, Kelekis N, Armonis V, Kouloulias V: Brachytherapy for prostate cancer: a systematic review. Adv Urol 2009:327945, Epub September 1, 2009.

-3 Brenner DJ, Curtis RE, Hall EJ, Ron E: Second malignancies in prostate carcinoma patients after radiotherapy compared surgery. Cancer 2000;88:398-406.

4 Mavrogenis AF, Pala E, Guerra G, Ruggieri P: Post-radiation sarcomas. Clinical outcome of 52 patients. J Surg Oncol 2012;105:570-576.

5 Gladdy RA, Qin LX, Moraco N, Edgar MA, Antonescu CR, et al: Do radiation-associated soft tissue sarcomas have the same prognosis as sporadic soft tissue sarcomas? J Clin Oncol 2010;28:2064-2069.

-6 Inoue YZ, Frassica FJ, Sim FH, Unni KK, Petersen IA, et al: Clinicopathologic features and treatment of postirradiation sarcoma of bone and soft tissue. J Surg Oncol 2000;75:42-50.

-7 Rubino R, Shamsaldin A, Le MG, Labbe M, Guinebretiere JM, et al: Radiation dose and risk of soft tissue and bone sarcoma after breast cancer treatment. Breast Cancer Res Treat 2005;89:277-288.

-8 Huvos AG, Woodward HQ, Cahan WG, Higinbotham NL, Stewart FW, et al: Postradiation osteogenic sarcoma of bone and soft tissue: a clinicopathologic study of 66 patients. Cancer 1985;55:1244-1255.

Miedler JD, MacLennan GT: Leiomyosarcoma of the prostate. J Urol 2007;178:668.

10 Sexton WJ, Lance RE, Reyes AO, Pisters PW, Tu SM, et al: Adult prostate sarcoma: the M.D. Anderson Cancer Center Experience. J Urol 2001;166:521-525.

11 Cheville JC, Dundore PA, Nascimiento AG, Meneses M, Kleer E, et al: Leiomyosarcoma of the prostate. Report of 23 cases. Cancer 1995;76:1422-1427.

12 Ahlering TE, Weintraub P, Skinner DG: Management of adult sarcoma of the bladder and prostate. J Urol 1988;140:1397-1399.

13 Vandoros GP, Manolidis T, Karamouzis MV, Gkermpesi M, Lambropoulou M, et al: Leiomyosarcoma of the prostate: case report and review of 54 previously published cases. Sarcoma 2008;2008:458709, Epub November 18, 2008.

14 Le Cesne A, Judson I, Crowther D, Rodenhuis S, Keizer HJ, et al: Randomized phase III study comparing conventional-dose doxorubicin plus ifosfamide versus high-dose doxorubicin plus ifosfamide plus recombinant human granulocyte-macrophage colony-stimulating factor in advanced soft tissue sarcomas: a trial of the European Organization for Research and Treatment of Cancer/Soft Tissue and Bone Sarcoma Group. J Clin Oncol 2000;18:2676-2684.

15 Pervaiz N, Colterjohn N, Farrokhyar F, Tozer R, Figueredo A, et al: A systematic meta-analysis of randomized controlled trials of adjuvant chemotherapy for localized resectable soft-tissue sarcoma. Cancer 2008;113:573-581.

$\checkmark 16$ Tseng TY, Sevilla DW, Moul JW, Maloney KE: Prostatic carcinosarcoma 15 years after combined external beam radiation and brachytherapy for prostatic adenocarcinoma: a case report. Prostate Cancer Prostatic Dis 2006;9:195-197.

-17 Scully JM, Uno JM, McIntyre M, Mosely S: Radiation-induced prostatic sarcoma: a case report. J Urol 1990;144:746-748.

18 Dominguez A, Piulatsb JM, Suarez JF, Condom E, Castells M, et al: Prostatic sarcoma after conservative treatment with brachytherapy for low-risk prostate cancer. Acta Oncol 2013;52:1215-1216. 


\section{Case Reports in Oncology}

\begin{tabular}{l|l}
\hline Case Rep Oncol 2014;7:565-570 \\
\hline DOI: $10.1159 / 000366294$ & $\begin{array}{l}@ 2014 \text { S. Karger AG, Basel } \\
\text { www.karger.com/cro }\end{array}$ \\
\hline
\end{tabular}

Horiguchi et al.: Radiation-Induced Leiomyosarcoma of the Prostate after Brachytherapy for Prostatic Adenocarcinoma

19 Gonin-Laurent N, Gibaud A, Huygue M, Lefevre SH, Le Bras M, et al: Specific TP53 mutation pattern in radiation-induced sarcomas. Carcinogenesis 2006;27:1266-1272.

20 Gonin-Laurent N, Hadj-Hamou NS, Vogt N, Houdayer C, Gauthiers-Villars M, et al: RB1 and TP53 pathways in radiation-induced sarcomas. Oncogene 2007;26:6106-6112.
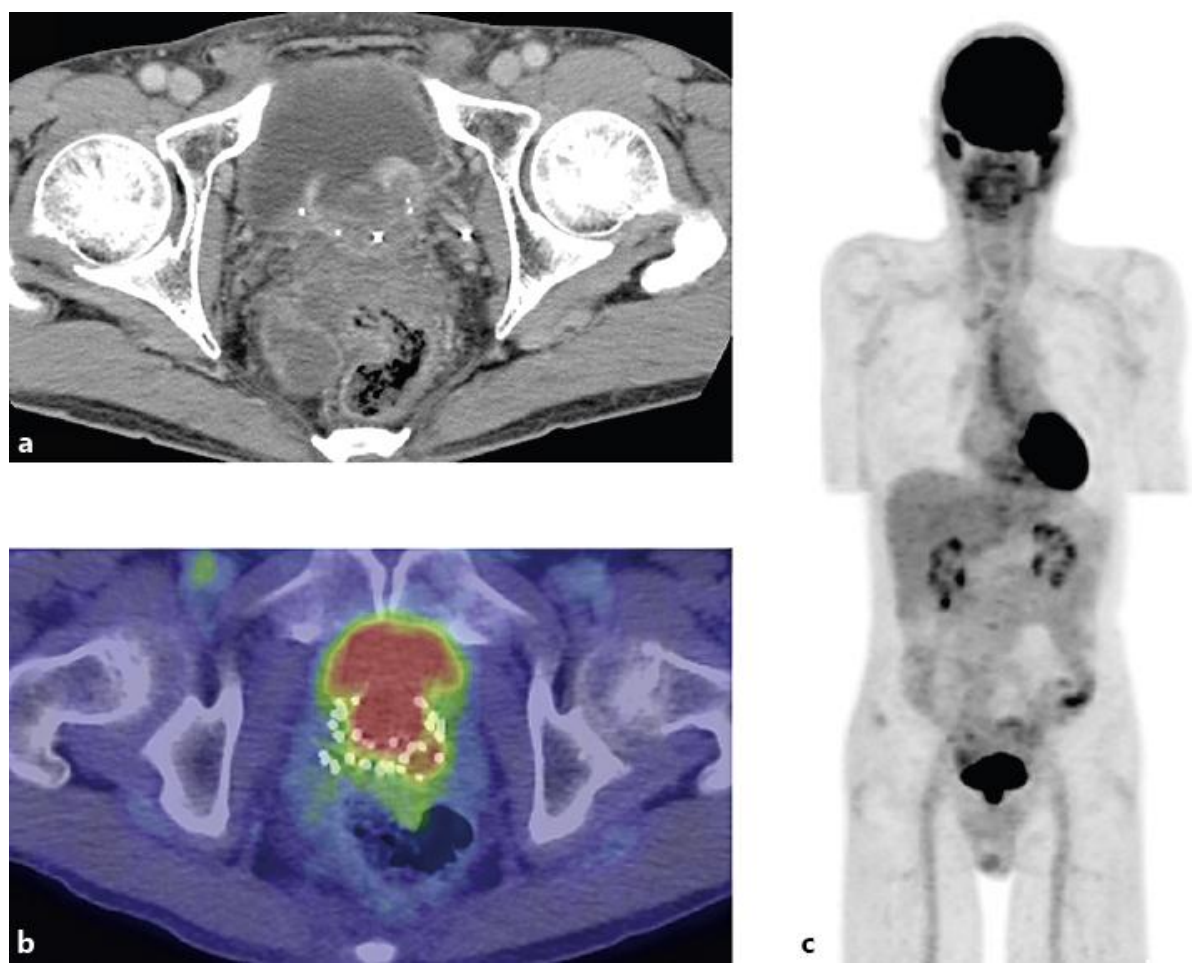

Fig. 1. a Computed tomography showing an enlarged prostate of about $5 \mathrm{~cm}$ with high density of metallic seeds. Tumor invaded into the inferior of the bladder without metastatic lesion. Positron emission tomography (b, c) showing a prostatic tumor with increased abnormal uptake. No other abnormal uptake was found. 


\section{Case Reports in Oncology}

\begin{tabular}{l|l}
\hline Case Rep Oncol 2014;7:565-570 \\
\hline DOI: 10.1159/000366294 & $\begin{array}{l}\text { C 2014 S. Karger AG, Basel } \\
\text { www.karger.com/cro }\end{array}$ \\
\hline
\end{tabular}

Horiguchi et al.: Radiation-Induced Leiomyosarcoma of the Prostate after Brachytherapy for Prostatic Adenocarcinoma

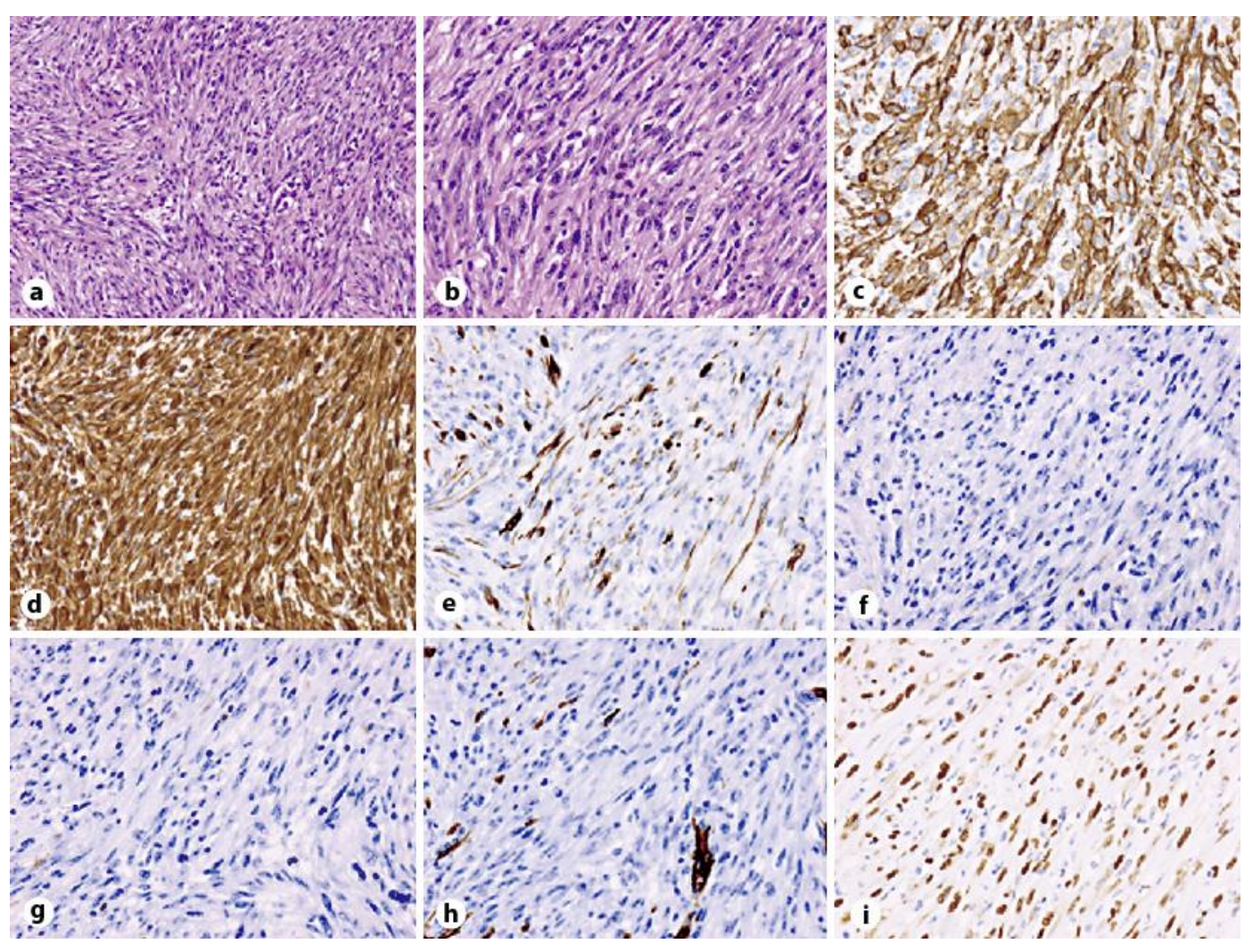

Fig. 2. Microscopic findings from a resected specimen. HE staining showing tumor cells with fascicles of atypical spindle-shaped cells $(\mathbf{a} \times 100)$, and strongly atypical clubbed nuclei and acidophilic cytoplasm (b $\times 200$ ). Immunohistochemistry showing tumor cells positive for SMA (c), vimentin (d), and desmin (e) and negative for S-100 (f), CD117 (g), and CD34 (h). Tumor cells overexpressed p53 (i). 